\title{
Numerical Analysis on the Structure Type and Mechanical Response of Tunnel Crossing Active Reverse Fault
}

\author{
Xianmin $\operatorname{Han}^{1,2}$ and Wenjiang $\operatorname{Li} \mathbb{D}^{1,2}$ \\ ${ }^{1}$ School of Civil Engineering, Shijiazhuang Tiedao University, Shijiazhuang 050043, China \\ ${ }^{2}$ State Key Laboratory of Mechanical Behavior and System Safety of Traffic Engineering Structures, Shijiazhuang Tiedao University, \\ Shijiazhuang 050043, China
}

Correspondence should be addressed to Wenjiang Li; liwenjiang@stdu.edu.cn

Received 14 January 2021; Accepted 9 August 2021; Published 19 August 2021

Academic Editor: Stefano Lo Russo

Copyright (c) 2021 Xianmin Han and Wenjiang Li. This is an open access article distributed under the Creative Commons Attribution License, which permits unrestricted use, distribution, and reproduction in any medium, provided the original work is properly cited.

Faulting would result in ground deformation and even damage to the tunnel structure. Thus, special structural designs should be made when the tunnel passes through an active fault. A single-track railway tunnel crossing a reverse fault is used to study the suitable structure type. In this paper, antidislocation structural measures such as reasonable segment length of articulated lining, cross-section shape, and thickness of lining are discussed through the numerical simulation. Firstly, the rational segment length of the articulated lining is confirmed. Stress and deformation behavior of articulated lining are also analyzed after the fault move. Then, the antifault effect of two kinds of cross-section shapes and three different lining thicknesses are compared. Researches show that the segment length of the articulated lining could be confirmed by the longitudinal distribution of maximum bending moment of lining after faulting. There are apparent stress concentrations occurring in the lining segment crossing the fault plane, and serious damage appears in the vault and wall waist of the tunnel. The horseshoe section with big curvature inverted arch is recommended to a single-track railway tunnel across an active fault. Simply increasing lining thickness is not suggested in a tunnel structure design crossing the active fault. The size of tunnel expansion and the minimum length of segment across fault plane can be geometrically determined according to fault dislocation magnitude and dip angle.

\section{Introduction}

With the continuous expansion of China's infrastructure construction scale and the implementation of the western development strategy, some highway, railway, and subway tunnels would inevitably pass through the seismically active zones.

The motion of an active fault includes the stick-slip and creep-slip mode. The rapid rupture of the fault is a kind of stick-slip dislocation, which is often accompanied by an earthquake and results in surface rupture zones and surface displacements. Slowly faulting without earthquake occurring is called a creep-slip dislocation, which also can lead to surface rupture and displacement [1]. The effect of fault reactivation on the tunnel are mainly manifested as structural damage caused by rock mass vibration (i.e., seismic problem) and the destruction caused by faulting slowly (i.e., anti- faulting problem). Past earthquakes show that faulting is the main cause of tunnel failure in comparison with seismic excitation.

During the planning phase of a tunnel project, the active fault zone should be avoided as far as possible. Special designs should be carried out in case the fault cannot be circumvented. At present, the antifaulting measures of the tunnel structure mainly include flexible joints design, setting cushioning, and damping layer, enlarging the excavation cross-section design. Some tunnel projects have adopted the typical antifaulting design, and scholars have conducted some relative research.

In order to reduce the damage of tunnel induced by faulting, partition measures are adopted in the tunnel lining design. The articulated lining is a special partition structure. It was utilized in the Bolu road tunnel in Turkey, Koohrang- 
III water transfer tunnel in Iran, etc. while crossing the active faults [2-7]. In the Xi'an subway tunnel design, segmental joints and enlarging section measures were used to withstand the large deformation of ground fissures [8].

Numerical modeling is an important design tool to disclose the soil-structure interaction between the tunnel lining and the surrounding soil. Many researchers and practitioners use finite element (FE) methods [9-15], or finite difference (FD) methods [16-17] to perform numerical modeling for tunnels.

Among them, the effects of tunnel position, fault displacement, crossing angle, and fault dip angle on the continuous tunnel have been studied $[14,15]$. The faulting effect on twin tunnels built by different construction methods was investigated $[9,10]$.

As for the laboratory test, a centrifuge test is a common means used to study the faulting effect on the tunnel. The authors [18] conducted the first centrifuge physical modeling to assess the interaction of a continuous tunnel at the intersection with active faults. A series of centrifuge tests are planned and tested on continuous tunnels by Baziar et al. [19]. Kiani et al. [20, 21] studied the behavior of a tunnel crossing with a normal fault and evaluated the behavior of the shallow tunnels affected by a normal fault with different dip angles using centrifugal laboratory models. Failure mechanism, progression, and locations of damages to the continuous tunnels are assessed through a gradual increase in ground displacement [22-29].

Although the damage characteristics of the lining can be reproduced more truly by centrifugal test, it has some problems such as high cost, consistency between prototype and model, and boundary problems.

Due to the complex mechanical response of the tunnel, the segment length, the cross-section shape of the tunnel, and its stress characteristics while faulting still need to be further studied. Taking a single-line railway tunnel as an example, the reasonable segment length of the articulated lining is firstly determined. Then, the stress distribution and deformation characteristics of the articulated structure are analyzed under faulting through numerical calculation. Comparative studies are also made on the antifaulting effect of different cross-section shapes and lining thickness. Finally, the overcut design of the tunnel section is discussed. Those would provide a technical reference for the design of tunnel structure across active faults.

\section{Materials and Methods}

2.1. Study on the Segment Length of the Lining Structure. For the tunnel located in an active fault zone, the tunnel lining would suffer great bending and distortion under the action of the strong faulting force. The conventional composite lining type has difficulty withstanding such strong external force; hence, lining damage and structural failure would appear. To minimize the damage caused by fault dislocation, flexible joints are often used in the tunnel lining design. Therefore, the continuous lining is divided into small segments by flexible joints. The deformation and stress concentration mainly occur in the segment connection part with

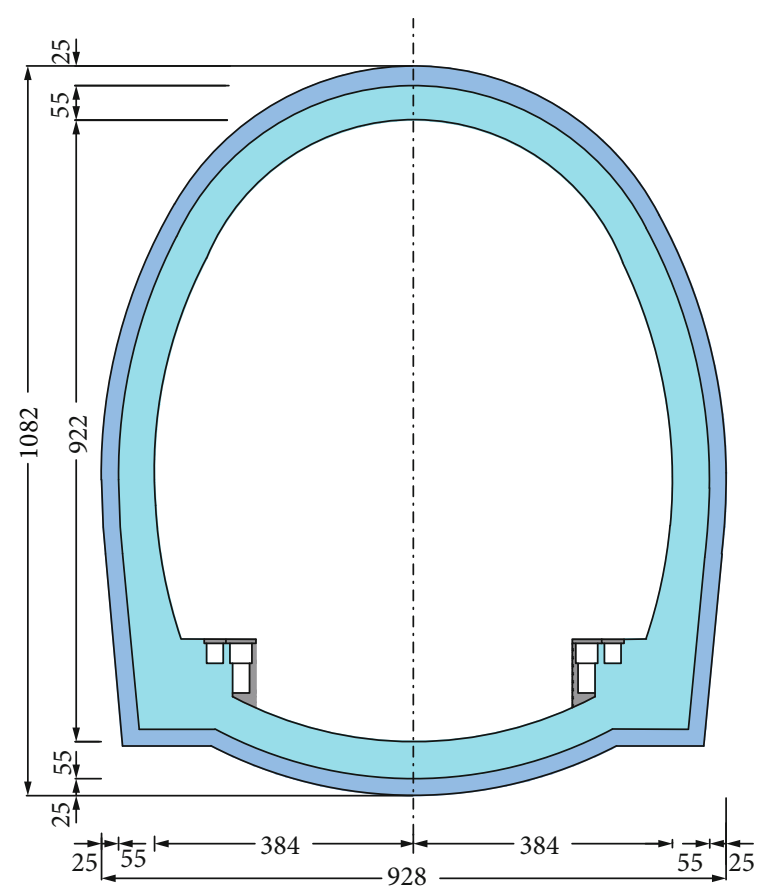

FIGURE 1: Horseshoe section of tunnel lining (units: $\mathrm{cm}$ ).

less stiffness and greater flexibility when faulting. As a result, the integrated damage of the structure is avoided.

In this paper, finite element numerical calculation is used to determine the reasonable segment length of tunnel lining according to the bending moment distribution characteristics. The dip angle of the fault is $70^{\circ}$, and the slip distance is set as $20 \mathrm{~cm}$. The horseshoe section shape of the single-line railway tunnel utilized in the numerical calculation is shown in Figure 1, and the main mechanical indexes of the lining are shown in Table 1. The lining material follows the Mohr-Coulomb yield criterion.

2.2. Method to Reveal the Stress and Deformation Characteristics of Lining under Fault Dislocation. To study the mechanical response of the articulated lining tunnel crossing an active fault, a 3D numerical calculation model (Figure 2) is used. The numerical model is $50 \mathrm{~m}$ long, $56 \mathrm{~m}$ wide, and $62 \mathrm{~m}$ high. The buried depth of the tunnel is $24 \mathrm{~m}$. The dip angle of the fault is $70^{\circ}$. Eight-node hexahedron elements are used to simulate the surrounding rock, primary support, and lining of the tunnel. The model is divided into 303852 elements. The interface is used to simulate the fault plane. The segment length of the tunnel is $14 \mathrm{~m}$, and one of the segments is intersecting with the fault plane. The width of the link section, namely, the special deformation joint, is $10 \mathrm{~cm}$. The filling material of deformation joints is an elastic material low-pressure polyethylene which is commonly used.

In the numerical calculation model, the rock mass, primary support, and the secondary lining are all elastoplastic material following the Mohr-Coulomb yield criterion. The filling material of deformation joints is treated as the 
TABLE 1: Mechanical parameters of the lining used in numerical calculation.

\begin{tabular}{lcccc}
\hline Lining & EA $(\mathrm{kN})$ & $N_{\mathrm{p}}(\mathrm{kN})$ & $\mathrm{EI}\left(\mathrm{kN} \cdot \mathrm{m}^{2}\right)$ & $M_{\mathrm{p}}(\mathrm{kN} \cdot \mathrm{m})$ \\
\hline C45 reinforced concrete $(55 \mathrm{~cm}$ thick) & $5.786 \times 10^{8}$ & 48555 & $5.918 \times 10^{9}$ & $1.40 \times 10^{5}$ \\
\hline
\end{tabular}

EA presents tension and compression stiffness in the axial direction; $N_{\mathrm{p}}$ presents maximum tensile resistance; EI presents flexural rigidity; and $M_{\mathrm{p}}$ presents the maximum bending moment.

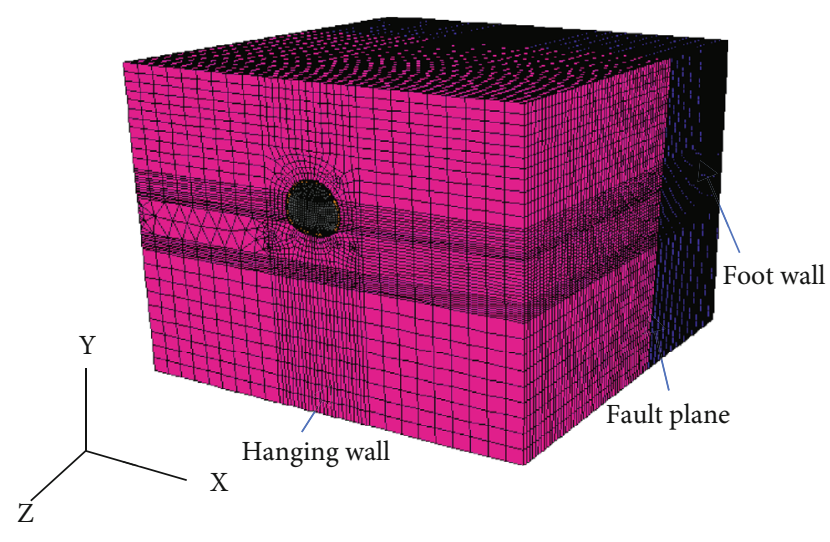

FIgURE 2: 3D numerical calculation model.

linear elastic constitutive model. The physical-mechanical parameters used in the numerical calculation are shown in Table 2.

The forced displacement method is used to simulate the fault dislocation. Faulting simulation includes two steps. The first is the generation of the initial stress of lining. In this step, the boundary of the upper surface of the model is free, and the other boundaries are normal constraints. The second is reverse fault dislocation. The boundaries of the footwall remain unchanged, and the lateral and bottom boundaries of the hanging wall are replaced by the displacement vector. The vector is upwards and parallels to the fault plane. The faulting value is $20 \mathrm{~cm}$.

2.3. Research on the Applicability of Section Shape. To find a reasonable cross-section shape for the tunnel across the active fault, two different kinds of section shapes are selected to be compared. The section forms are separately horseshoe and circular. The two sections are area equivalent. The horseshoe section is shown in Figure 1. The inner contour area of the horseshoe section is $55.96 \mathrm{~m}^{2}$, so the outer radius of the circular section should be $4.22 \mathrm{~m}$. The supporting parameters of the two sections are the same. The lining thickness of the circular section is also $55 \mathrm{~cm}$. Stress and deformation differences between the different section shapes are analyzed using numerical simulation.

2.4. Research on the Influence of Thickness of the Segmental Lining. The stiffness of a tunnel structure is mainly affected by material property and lining thickness. Under the action of external force, the stiffness has a great influence on the deformation of the structure. To study the influence of cross-section size on the structure stress and deformation under fault dislocation, three different lining thicknesses are chosen. The lining thicknesses are separately $30 \mathrm{~cm}$, $55 \mathrm{~cm}$, and $85 \mathrm{~cm}$. Numerical simulation is adopted to analyze the mechanical effect of different lining thicknesses while faulting.

2.5. Design Method of Lining Structure Redundancy. During fault dislocation, the deformation of the lining segment intersecting with the fault plane is more complex. Translation movement and rigid body deflection of the segment occur at the same time. Because the stress concentration level of this segment is high, its damage is the most serious among all segments. Therefore, in the length design of the lining segment, a lining segment should be set across the fault plane to reduce the influence of dislocation on adjacent segments.

Because the tunnel lining could not resist the huge force caused by fault dislocation, it is usually adopted to enlarge the excavation section of the tunnel to provide the space for the repair and maintenance of the tunnel. While reverse fault dislocating, the tunnel design idea is shown in Figure 3. Taking the inner clear height $(H)$ of the tunnel lining as the controlling index, the inner clear loss of the tunnel is $\Delta h 1$ ( $\Delta h 1=S \sin \alpha$ when the fault dislocation is $S$. Therefore, the enlarged section size of the tunnel lining in the uplifted wall should be $D(D=H+S \sin \alpha)$ through expanding excavation of the tunnel bottom. In terms of geometric concepts, the minimum length of the lining segment intersecting the fault plane is $L(L=H(H+S \sin \alpha) \cot \alpha=H \cot \alpha+S \cos \alpha$ ). Thus, the tunnel redundancy (or expanding excavation size) is determined by fault slip magnitude and dip angle. And the minimum length of lining segment intersecting with the fault plane is determined by the inner clearance of lining, fault slip magnitude, and dip angle.

\section{Results and Discussion}

3.1. Determination of the Suitable Segment Length of Lining Structure. The bending moment distribution of the tunnel lining after faulting is shown in Figure 4 . The value of the maximum bending moment is $30.69 \times 10^{3} \mathrm{kN} \cdot \mathrm{m}$. It can be concluded that the maximum bending moment of the lining appears on both sides of the fault, and the length between them is $14 \mathrm{~m}$. In the articulated design, the failure of tunnel lining is mainly controlled by the maximum bending moment. Since the deformation joints in the lining structure are flexible connections, its design value of bending moment should be less than that of the lining. That could ensure that the flexible connection should be destroyed before the lining segment destruction. The flexible connection parts become plastic hinges. Therefore, in the design of the lining, the segment length should be less than the distance between the 
TABLE 2: Equivalent physical and mechanical indexes adopted in the numerical model.

\begin{tabular}{lcccccc}
\hline Items & $\begin{array}{c}\text { Unit weight } \\
\left(\mathrm{kN} \cdot \mathrm{m}^{-3}\right)\end{array}$ & $\begin{array}{c}\text { Elasticity modulus } \\
(\mathrm{GPa})\end{array}$ & $\begin{array}{c}\text { Poisson's } \\
\text { ratio }\end{array}$ & $\begin{array}{c}\text { Cohesion } \\
(\mathrm{MPa})\end{array}$ & $\begin{array}{c}\text { Internal friction } \\
\text { angle }\left(^{\circ}\right)\end{array}$ & $\begin{array}{c}\text { Tensile strength } \\
(\mathrm{MPa})\end{array}$ \\
\hline Rock mass of hanging wall & 22 & 3.5 & 0.33 & 0.45 & 33 & 0.168 \\
Rock mass of foot wall & 19 & 1.5 & 0.38 & 0.15 & 25 & 0.047 \\
Preliminary support & 25 & 29.86 & 0.2 & 1.8 & 60 & 60 \\
Lining & 25 & 34.19 & 0.2 & 2.35 & - & - \\
Material of deformation joint & 10 & 0.006 & 0.42 & - & - \\
\hline
\end{tabular}

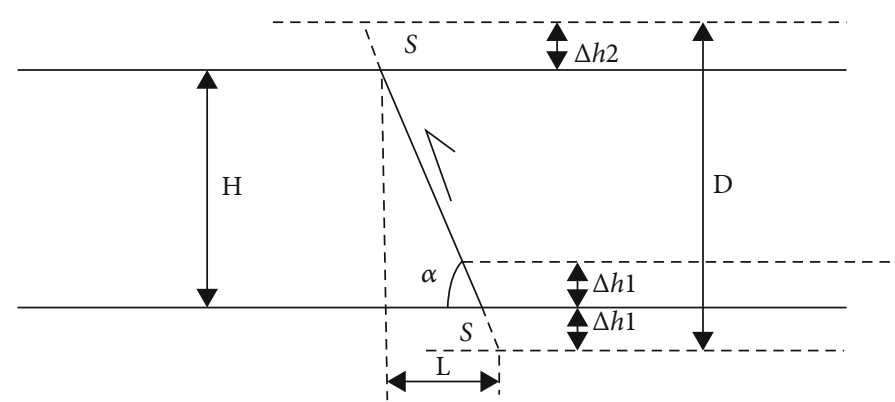

FIgURE 3: Diagram of clearance change of the tunnel lining segment under reverse fault dislocation.

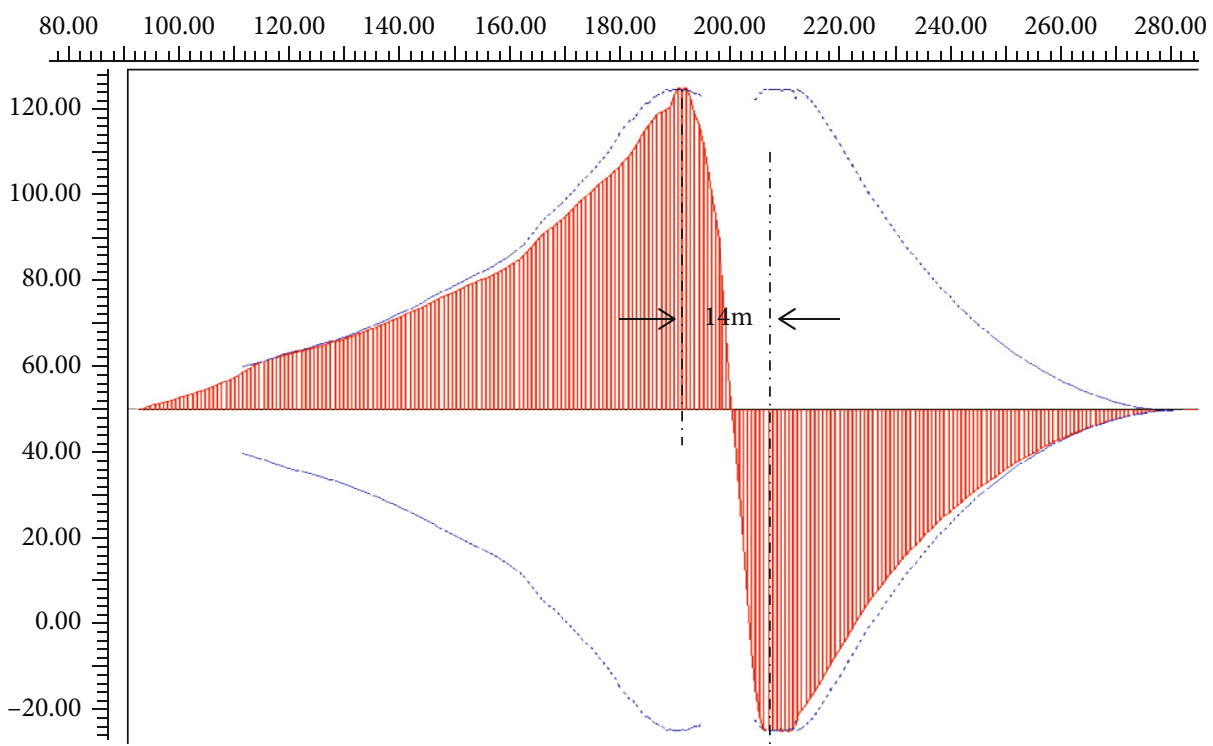

FIGURE 4: Bending moment diagram of lining after fault dislocation.

two maximum moment locations. The maximum segment length of the lining should be $14 \mathrm{~m}$.

\subsection{Stress and Deformation Characteristics of the Segmental Lining under Fault Dislocation}

(1) Stress distribution characteristics of the lining

After the dislocation of the reverse fault reaches $20 \mathrm{~cm}$, the maximum and minimum principal stress and maximum shear stress of the segments are shown in Figures 5-7. Con- sidering the boundary effect of the numerical model, the stress characteristics of the middle three segments (namely, segment 2, segment 3, and segment 4) are analyzed.

(1) Distribution of the maximum principal stress

Figure 5 indicates that the maximum tensile stress of segment 2 in the footwall (fixed part) is $1.25 \mathrm{MPa}$ and appears at the waist and foot of the lining wall. The maximum tensile stress of segment 3 intersecting with the fault plane is $1.26 \mathrm{MPa}$ and occurs at the waist of the sidewall. 


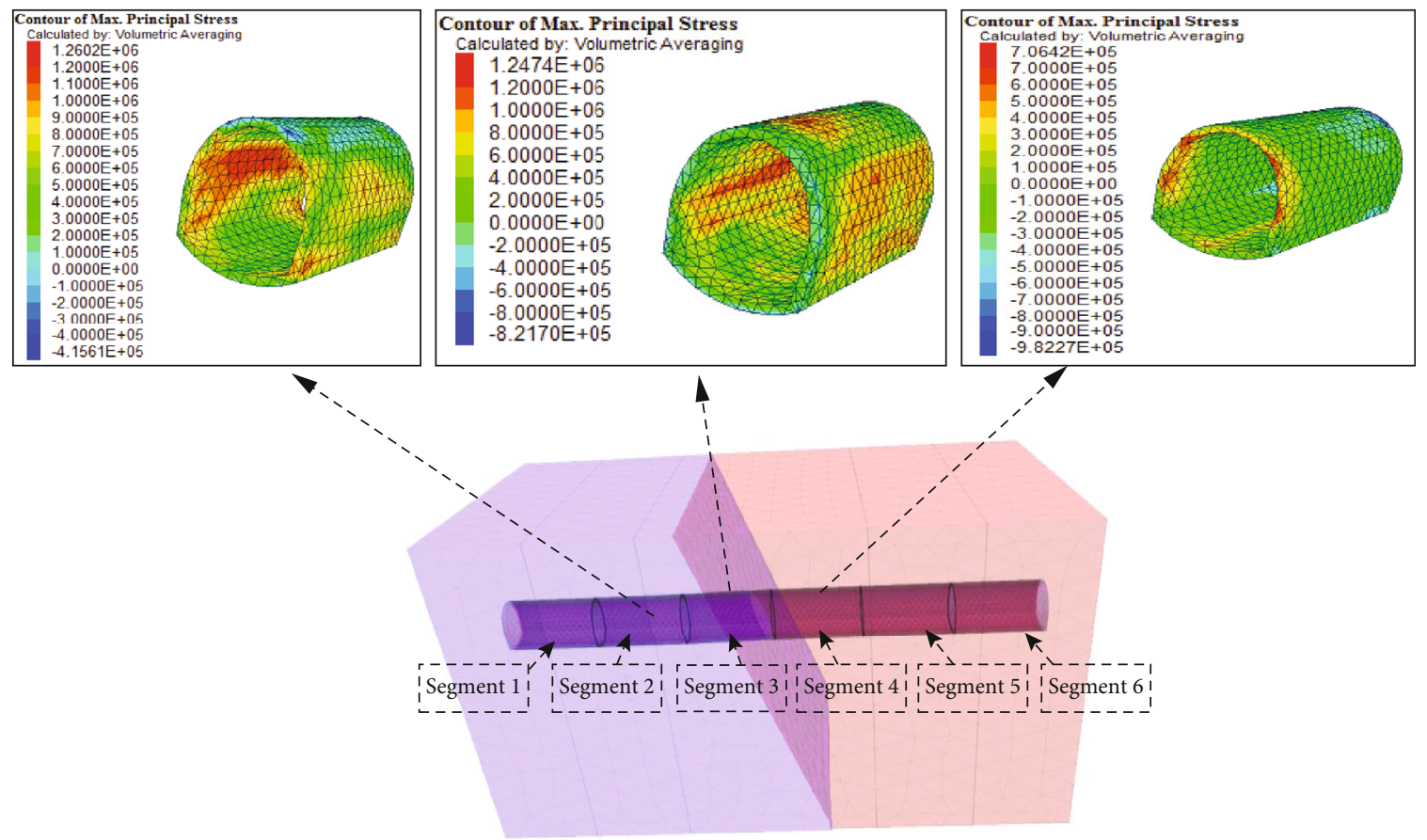

FIgURE 5: Maximum principal stress nephogram (units: Pa).
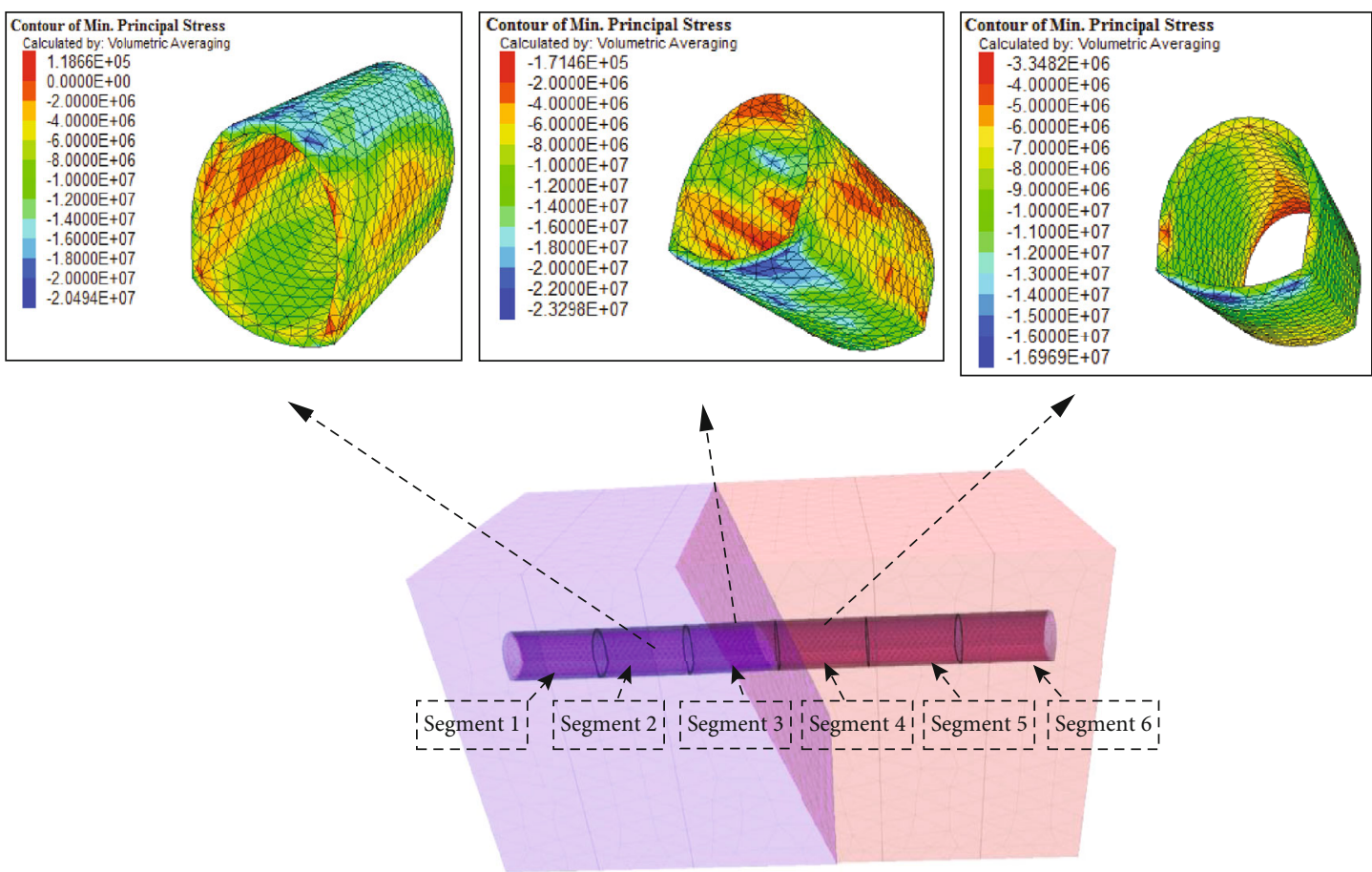

FIGURE 6: Minimum principal stress nephogram (units: Pa).

The maximum tensile stress of segment 4 in the hanging wall (rising part) is $0.71 \mathrm{MPa}$ and occurs at the waist of the sidewall. The maximum principal stress of segment 3 is almost 1.7 times as much as segment 4 .

Therefore, the tensile stress of the segments intersecting with the fault plane and lying in the footwall is larger, while the tensile stress of the segment in the hanging wall is smaller.
The maximum tensile stress occurs mainly at the waist of sidewall. The wall waist is prone to longitudinal cracks.

(2) Distribution of the minimum principal stress

According to Figure 6, the maximum compression stress of segment 2 in the footwall is $-20.5 \mathrm{MPa}$ and appears in the 

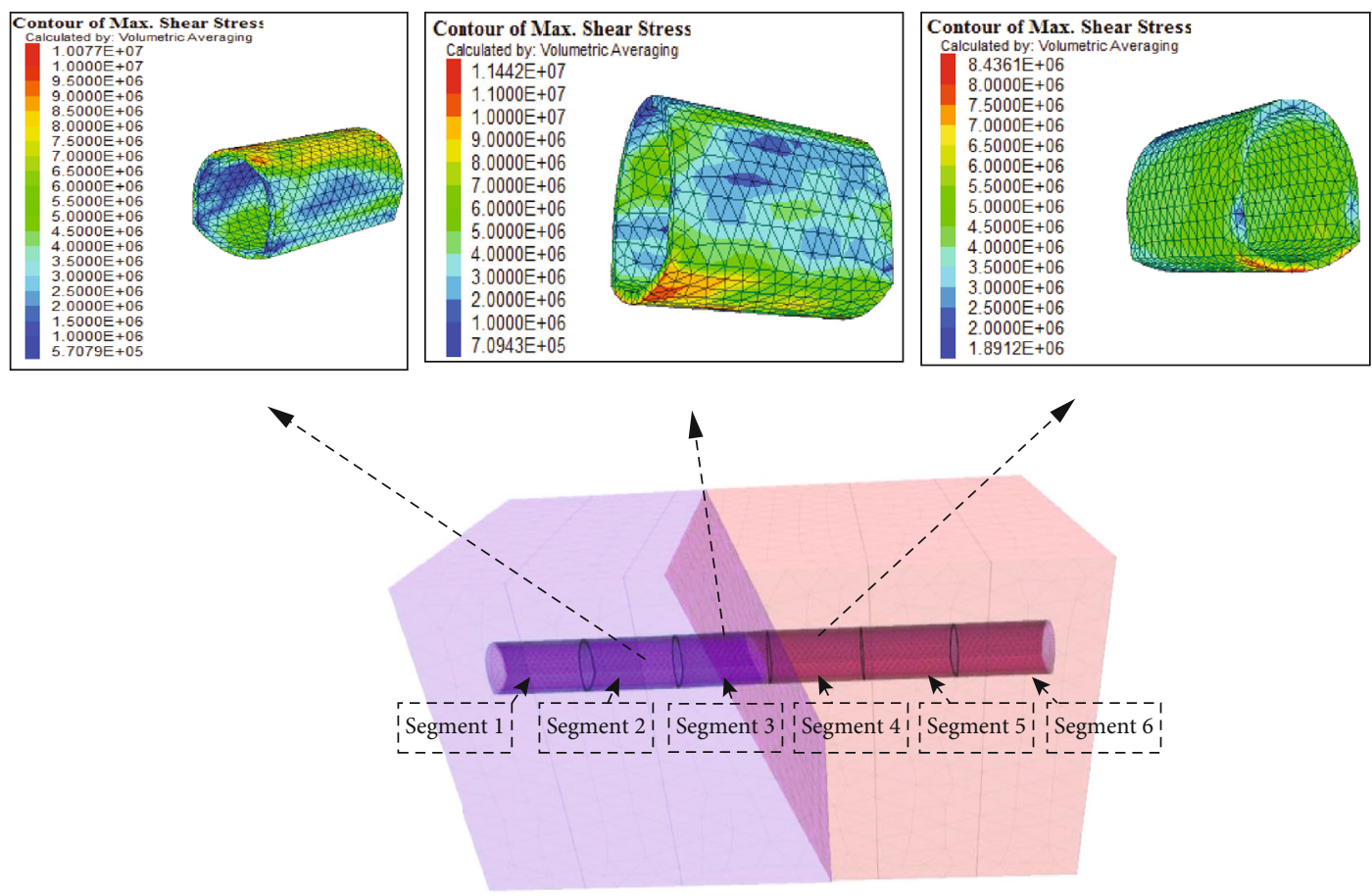

Figure 7: Maximum shear stress nephogram (units: Pa).

arch of the tunnel. The maximum compression stress of segment 3 intersecting the fault plane is $-23.3 \mathrm{MPa}$ and occurs at the inverted arch of the tunnel. The maximum compression stress of segment 4 in the hanging wall is $-16.9 \mathrm{MPa}$ and occurs in the inverted arch of the tunnel. The minimum principal stress of segment 3 is almost 1.4 times as much as segment 4 .

Therefore, the compression stress of the segment intersecting with the fault plane is the greatest. Affected by the drag and squeeze action of the fault, the compression stress of the segments in the footwall is greater than that in the hanging wall. The maximum compression stress of the segments in the hanging wall and intersecting with the fault mainly occurred in the inverted arch of the tunnel. In contrast, the maximum compression stress of the segments in the footwall has mainly appeared in the arch of the tunnel.

\section{(3) Distribution of the maximum shear stress}

According to Figure 7, the maximum shear stress of segment 2 in the footwall is $10.7 \mathrm{MPa}$ and appears in the arch of the tunnel. The maximum shear stress of segment 3 intersecting with the fault plane is $11.4 \mathrm{MPa}$ and appears in the inverted arch of the tunnel. The maximum shear stress of segment 4 in the hanging wall is $8.4 \mathrm{MPa}$ and occurs in the inverted arch of the tunnel. The maximum shear stress of segment 3 is almost 1.4 times as much as segment 4 .

For the shear stress value, the biggest appears in the segment intersecting with the fault plane. And the stress of the segment in the footwall is bigger than that in the hanging wall. The maximum shear stress of the segments in the footwall and intersecting with the fault occurs mainly in the

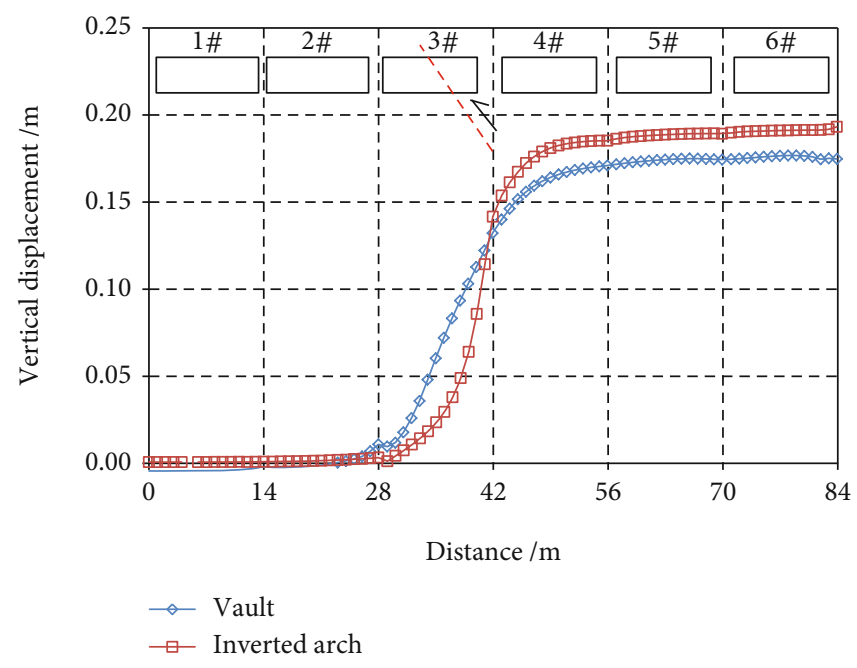

FIgURE 8: Deformation curve of segmental lining under fault dislocation.

inverted arch. The maximum shear stress of the segment in the hanging wall is mainly concentrated in the vault of the tunnel.

(2) Deformation characteristics of the lining

Due to the faulting, the tunnel lining is subjected to forced displacement resulting in significant vertical deformation. Therefore, two typical positions of the vault and the bottom of the inverted arch are selected to monitor the lining displacement during the faulting. Figure 8 indicates the vertical displacement curves of typical positions. 
TABLE 3: Comparison of lining stress between circular and horseshoe section shapes.

\begin{tabular}{lccccccccccc}
\hline & & \multicolumn{3}{c}{ Segment 2 } & \multicolumn{3}{c}{ Segment 3 } & \multicolumn{3}{c}{ Segment 4 } \\
Items & & $\sigma_{\max }(\mathrm{MPa})$ & $\begin{array}{c}\sigma_{\min } \\
/ \mathrm{MPa}\end{array}$ & $\begin{array}{c}\sigma_{\text {shear }} \\
(\mathrm{MPa})\end{array}$ & $\begin{array}{c}\sigma_{\max } \\
(\mathrm{MPa})\end{array}$ & $\begin{array}{c}\sigma_{\min } \\
(\mathrm{MPa})\end{array}$ & $\begin{array}{c}\sigma_{\text {shear }} \\
(\mathrm{MPa})\end{array}$ & $\sigma_{\max }(\mathrm{MPa})$ & $\begin{array}{c}\sigma_{\min } \\
(\mathrm{MPa})\end{array}$ & $\begin{array}{c}\sigma_{\text {shear }} \\
(\mathrm{MPa})\end{array}$ \\
\hline \multirow{2}{*}{ Circular } & Value & +1.49 & -16.8 & 8.7 & +2.12 & -20.4 & +10.9 & +0.99 & -13.0 & 6.9 \\
& Position & Top & Bottom & Bottom & Top & Bottom & Bottom & Top & Bottom & Bottom \\
\multirow{2}{*}{ Horseshoe } & Value & +1.26 & -20.5 & 10.7 & +1.25 & -23.3 & +11.4 & +0.71 & -16.9 & 8.4 \\
& Position & Inverted arch & Sidewall & Sidewall & Sidewall & Vault & Vault & Inverted arch & Vault & Vault \\
\hline
\end{tabular}

We made the following conclusions according to Figure 8: (1) The partition effect of deformation joints is obvious, and the vertical deformation of segments 1 and 2 located at footwall is very small. (2) The deformation of segment 3 intersecting the fault is more complex. Because of the great stiffness of the lining, a rigid deflection of segment 3 occurs besides upward displacement under the fault forced dislocation. (3) The deformation of the segments 4, 5, and 6 in the hanging wall is basically identical. However, the displacement of the vault is less than that of the inverted arch due to the constraint of the upper surrounding rock. Under the forced displacement applied to the bottom of the hanging wall, the rock mass between the bottom of the model and the tunnel in the hanging wall generates compression deformation. Therefore, the calculated displacement in the inverted arch of the tunnel is less than $20 \mathrm{~cm}$.

\subsection{The Applicability of Section Shape of the Tunnel Crossing an Active Fault}

(1) Comparison of stress characteristics of the lining segments

The calculation results of the maximum principal stress, minimum principal stress, and maximum shear stress of the main sections of different section shape tunnel lining are listed in Table 3.

The following can be seen from Table 3: (1) The maximum compression stress and shear stress of the circular shape are smaller than that of the horseshoe, while the maximum tensile stress is greater than that of the horseshoe after faulting. On a whole, the circular section has a better ability to withstand fault dislocation. (2) For the two kinds of crosssection shapes, the internal force of segment 3 crossing the fault plane is the largest and the damage is the most serious, followed by segment 2 in the footwall and segment 4 in the hanging wall. (3) The top and bottom of the circular shape tunnel bear big tensile stress, compression stress, and shear stress, which are the most vulnerable parts to damage.

(2) Comparison of deformation characteristics of lining segments

The vault and the bottom of the tunnel are selected to monitor the vertical deformation during the fault dislocation. The lining deformation of the horseshoe section is shown in Figure 8 above, and the deformation curve of the circular section is shown in Figure 9.

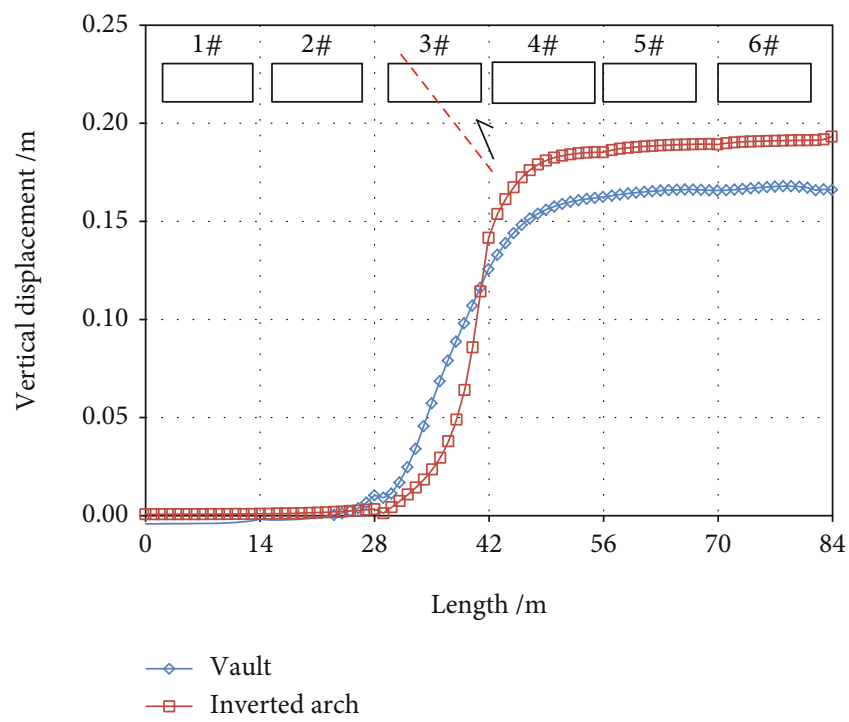

FIgURE 9: Lining deformation curve of the circular section under fault dislocation.

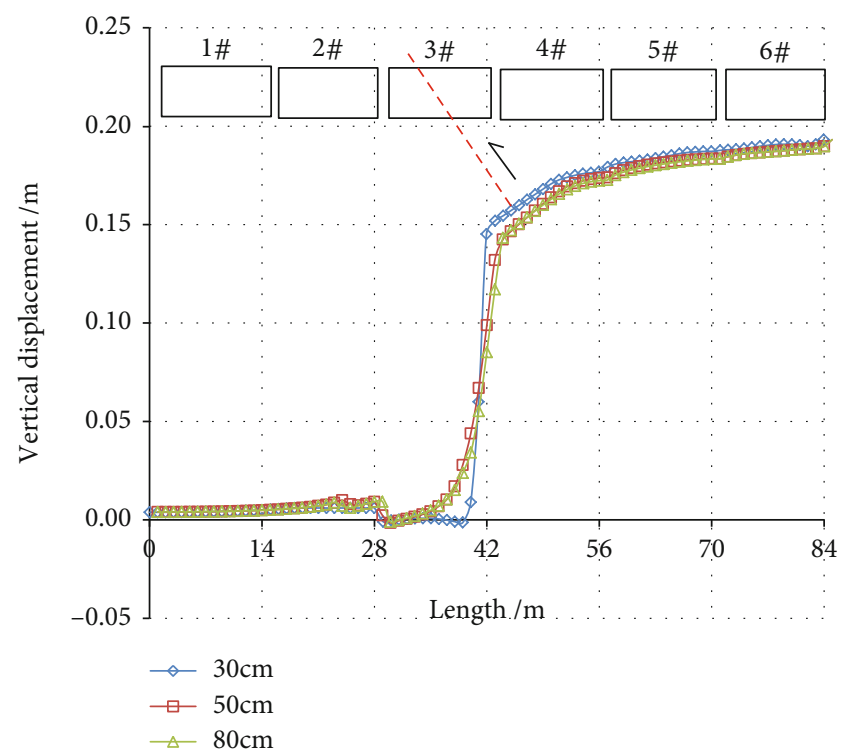

FIGURE 10: Vertical deformation curves of the inverted arch of different thicknesses under fault dislocation. 
TABLE 4: Comparison of segment stress under three different lining thicknesses.

\begin{tabular}{lccccccc}
\hline $\begin{array}{l}\text { Thickness } \\
\text { of lining }\end{array}$ & & Value & $\begin{array}{c}\sigma_{\max }(\mathrm{MPa}) \\
\text { Position }\end{array}$ & Value & $\begin{array}{c}\sigma_{\text {min }}(\mathrm{MPa}) \\
\text { Position }\end{array}$ & \multicolumn{2}{c}{$\sigma_{\text {shear }}(\mathrm{MPa})$} \\
Position
\end{tabular}

The following can be concluded: (1) The deformation characteristics of the circular and horseshoe segmental lining are the same when the reverse fault moves. (2) The vertical deformation of the inverted arch is the same. The vertical deformation of the circular vault is smaller than that of the horseshoe section, but the difference is small. The calculation results show that the circular section has some advantages over the horseshoe section in deformation resistance, but the difference is not significant.

In conclusion, adopting the circular section can reduce the stress of the lining to a certain extent, but it has no obvious advantages in resisting dislocation deformation. Considering the economy and the functional requirements of the single-track railway tunnel section, the horseshoe section with large curvature is recommended to increase the effective utilization rate of the section.

\subsection{Influence of Thickness of the Segmental Lining}

\section{(1) Deformation characteristics of the lining structure}

The deformation difference of the lining with three kinds of section thicknesses is illustrated by the vertical deformation of the inverted arch, as shown in Figure 10. It indicates that the deformation characteristics of lining with different section thicknesses have no obvious difference.

\section{(2) Mechanical characteristics of the lining structure}

The statistics of the stress of lining segments near the fault plane are listed in Table 4.

The following conclusions are obtained: (1) Under different lining thicknesses, the maximum value of principal stress and shear stress all appeared in segment 3 across the fault, followed by segment 4 in the hanging wall. The least value appears in segment 2. (2) When the thickness of the lining changes, the larger the section thickness is, the greater the minimum principal stress, and the maximum shear stress are. However, the variation of maximum principal stress with the section thickness is not apparent. (3) It is not recommended to simply increase the lining section thickness to resist fault dislocation.
3.5. Design of Lining Structure Redundancy. For the tunnel engineering, the clearance height $H$ of the tunnel lining is $9.22 \mathrm{~m}$, and the dip angle of the reverse fault is $70^{\circ}$. When the dislocation of fault is $20 \mathrm{~cm}$, the tunnel needs to be expanded by $0.19 \mathrm{~m}$, and the section clearance height after the expansion is $9.41 \mathrm{~m}$ by the design method mentioned above. From a geometric point of view, the minimum length of the lining segment intersecting the fault plane is $3.5 \mathrm{~m}$.

\section{Conclusions}

For a tunnel across an active reverse fault, the reasonable segment length of the articulated lining, the effect of the lining section shape and size, the expanding excavation size of the tunnel section, and mechanical response are all studied using numerical simulation. The main conclusions are drawn as follows:

(1) The maximum segment length of the lining can be determined according to the bending moment distribution of the lining after fault dislocation. Under the engineering conditions abovementioned, the segment length should less than $14 \mathrm{~m}$

(2) When the fault moves, the tensile stress, compression stress, and shear stress of the lining segment intersecting with the fault are the largest. And its stress value is $1.4 \sim 1.7$ times as much as the segment in the hanging wall. The vault and wall waist of the tunnel would be seriously damaged

(3) The circular section shape can effectively reduce the stress of lining structure, but it has no obvious advantage in resisting dislocation deformation. For the single-track railway tunnel, a horseshoe section shape with large curvature inverted arch is recommended to increase the effective utilization ratio of the section

(4) As the thickness of the lining increases, the structural stiffness increases, and the stress of the structure becomes larger when the fault moves. Thus, it is 
not suitable to adopt the measure of simply increasing the lining thickness to resist the fault dislocation

(5) The expanding excavation size of the tunnel section and the minimum length of lining segment across the fault plane can be determined according to the geometric characteristics of lining deformation during fault dislocation

\section{Data Availability}

The test data used to support the findings of this study are included within the article. Readers can obtain data supporting the research results from the test data table in the paper.

\section{Conflicts of Interest}

The authors declare no conflict of interest regarding the publication of this paper.

\section{Authors' Contributions}

The first author is Xian-min Han.

\section{Acknowledgments}

This study was supported by the State Key Laboratory for Track Technology of High-speed Railway of China Academy of Railway Sciences (Grant no. 2019YJ196). The support is very gratefully acknowledged.

\section{References}

[1] G. Lin, "Seismic analysis of underground structure," World Seismic Engineering, vol. 6, 1990.

[2] M. Russo, G. Germani, and W. Amberg, "Design and construction of large tunnel through active faults: a recent application," in International Conference of Tunnelling \& Underground Space Use, pp. 16-18, Istanbul, Turkey, 2002.

[3] A. Walter and R. Marco, "Seismic design of underground structures-the Bolu Tunnel," in Proceedings of the AITES-ITA 2001 World Tunnel Congress, pp. 137-147, Milano, Italy, 2001.

[4] S. Dalgıç, "Tunneling in squeezing rock, the Bolu Tunnel, Anatolian Motorway, Turkey," Engineering Geology, vol. 67, no. 12, pp. 73-96, 2002.

[5] A. R. Shahidi and M. Vafaeian, "Analysis of longitudinal profile of the tunnels in the active faulted zone and designing the flexible lining (for Koohrang-III tunnel)," Tunnelling and Underground Space Technology, vol. 20, no. 3, pp. 213-221, 2005.

[6] R. Caulfield, D. S. Kieffer, D. F. Tsztoo, and B. Cain, "Seismic design measures for the retrofit of the Claremont Tunnel," in Jacobs Associates. Rapid Excavation and Tunneling Conference (RETC) Proceedings, pp. 1128-1138, San Francisco, CA, USA, 2005.

[7] P. Li, Research on Anti-Breaking Structural Design of the Highway Tunnel across Active Fault Zones, Chongqing Jiaotong University, Chongqing, 2009.

[8] Q. B. Huang, J. B. Peng, Q. Y. Wang, and Y. H. Gao, "Reserved displacements for anti-creak design of Netro Tunnel passing through active ground fissure zones," Chinese Journal of Rock Mechanics and Engineering, vol. 29, 2010.

[9] T. Gregor, B. Garrod, and D. Young, "Analyses of underground structures crossing an active fault in Coronado, California," Proceedings of the World Tunnel Congress, vol. 3, pp. 445-450, 2007.

[10] I. Anastasopoulos, N. Gerolymos, V. Drosos, T. Georgarakos, R. Kourkoulis, and G. Gazetas, "Behaviour of deep immersed tunnel under combined normal fault rupture deformation and subsequent seismic shaking," Bulletin of Earthquake Engineering, vol. 6, no. 2, pp. 213-239, 2008.

[11] G. Lanzano, E. Bilotta, and G. Russo, "Tunnels under seismic loading: a review of damage case histories and protection methods," in Strategies for Reduction of the Seismic Risk, pp. 65-74, Publisher StreGa, 2008.

[12] X. Luo and Z. Yang, "Finite element modeling of a tunnel affected by dislocation of faults," in Proceedings of 5th Asia Pacific Congress on Computational Mechanics \& 4th International Symposium on Computational Mechanics (APCOM \& ISCM),, Singapore, 2013.

[13] M. G. Varnusfaderani, A. Golshani, and R. Nemati, "Behavior of circular tunnels crossing active faults," Acta Geodynamica et Geromaterialia, vol. 12, no. 4, pp. 363-377, 2015.

[14] L. Xu and M. Lin, "Analysis of buried pipelines subjected to reverse fault motion using the vector form intrinsic finite element method," Soil Dynamics and Earthquake Engineering, vol. 93, pp. 61-83, 2017.

[15] A. G. Chermahini and H. Tahghighi, "Numerical finite element analysis of underground tunnel crossing an active reverse fault: a case study on the Sabzkouh Segmental Tunnel," Geomechanics and Geoengineering, vol. 14, no. 3, pp. 155-166, 2019.

[16] M. Corigliano, L. Scandella, C. G. Lai, and R. Paolucci, "Seismic analysis of deep tunnels in near fault conditions: a case study in Southern Italy," Bulletin of Earthquake Engineering, vol. 9, no. 4, pp. 975-995, 2011.

[17] Z. Z. Wang, Z. Zhang, and B. Gao, "Seismic behavior of the tunnel across active fault," in InProceedings of the 15th world conference on earthquake engineering, pp. 24-28, Lisbon, Portugal, 2012.

[18] P. B. Burridge, R. F. Scott, and J. F. Hall, "Centrifuge study of faulting effects on tunnel," Journal of Geotechnical Engineering, vol. 115, no. 7, pp. 949-967, 1989.

[19] M. Baziar, A. Nabizadeh, C. Jung Lee, and W. Yi Hung, "Centrifuge modeling of interaction between reverse faulting and tunnel," Soil Dynamics and Earthquake Engineering, vol. 65, pp. 151-164, 2014.

[20] M. Kiani, T. Akhlaghi, and A. Ghalandarzadeh, "Experimental modeling of segmental shallow tunnels in alluvial affected by normal faults," Tunnelling and Underground Space Technology, vol. 51, pp. 108-119, 2016.

[21] M. Sabagh and A. Ghalandarzadeh, "Centrifuge experiments for shallow tunnels at active reverse fault intersection," Frontiers of Structural and Civil Engineering, vol. 14, no. 3, pp. 731-745, 2020.

[22] Y. G. Zhang, J. Tang, R. P. Liao et al., "Application of an enhanced BP neural network model with water cycle algorithm on landslide prediction," Stochastic Environmental Research and Risk Assessment, vol. 35, no. 6, pp. 1273-1291, 2021.

[23] Y. G. Zhang, J. Tang, Z. Y. He, J. K. Tan, and C. Li, “A novel displacement prediction method using gated recurrent unit 
model with time series analysis in the Erdaohe landslide," Natural Hazards, vol. 105, no. 1, pp. 783-813, 2021.

[24] Y. G. Zhang and L. N. Yang, "A novel dynamic predictive method of water inrush from coal floor based on gated recurrent unit model," Natural Hazards, vol. 105, no. 2, pp. 20272043, 2021.

[25] Y. G. Zhang, Z. Zhang, S. Xue, R. Wang, and M. Xiao, "Stability analysis of a typical landslide mass in the Three Gorges Reservoir under varying reservoir water levels," Environmental Earth Sciences, vol. 79, no. 1, 2020.

[26] Y. G. Zhang, S. Y. Zhu, J. K. Tan, L. D. Li, and X. J. Yin, “The influence of water level fluctuation on the stability of landslide in the Three Gorges Reservoir," Arabian Journal of Geosciences, vol. 13, no. 17, 2020.

[27] Y. G. Zhang, S. Zhu, W. Zhang, and H. Liu, "Analysis of deformation characteristics and stability mechanisms of typical landslide mass based on the field monitoring in the Three Gorges Reservoir, China," Journal of Earth System Science, vol. 128 , no. 1, 2019.

[28] B. Gong, Y. J. Jiang, P. Yan, and S. Zhang, "Discrete element numerical simulation of mechanical properties of methane hydrate-bearing specimen considering deposit angles," Journal of Natural Gas Science and Engineering, vol. 76, p. 103182, 2020.

[29] B. Gong, Y. J. Jiang, and L. J. Chen, "Feasibility investigation of the mechanical behavior of methane hydrate- bearing specimens using the multiple failure method," Journal of Natural Gas Science and Engineering, vol. 69, p. 102915, 2019. 